\title{
Método de Ensaio para Avaliação do Desempenho Físico-mecânico de Copos Plásticos Descartáveis
}

\author{
Leda Coltro, Fábio G. Teixeira \\ CETEA - ITAL
}

\begin{abstract}
Resumo: Os copos plásticos descartáveis disponíveis no mercado brasileiro foram tendo sua massa reduzida ao longo dos anos, o que levou à necessidade de se usar dois copos para desempenhar a função de contenção de líquidos. Por este motivo, foi desenvolvido um estudo de avaliação do desempenho físico-mecânico de copos plásticos descartáveis de diversas capacidades volumétricas com a finalidade de gerar subsídios aos trabalhos de padronização deste produto no mercado brasileiro mediante o estabelecimento de requisitos mínimos de desempenho. Este artigo tem por objetivo apresentar os critérios adotados durante o desenvolvimento desta metodologia, bem como os requisitos mínimos sugeridos para este produto.
\end{abstract}

Palavras-chave: Copos plásticos, PS, PP, descartáveis, requisitos mínimos, resistência à compressão.

\section{Test Method for Mechanical Evaluation of One-way Plastic Drinking-Cups}

Abstract: The one-way plastic drinking cups available in the Brazilian market have had their mass constantly decreased over the years, making it necessary to use two cups for efficient liquid retention. We have therefore carried out an evaluation of mechanical properties of plastic cups of various sizes in order to establish minimum performance requirements to be used in standardization of this product in the Brazilian market. The objective of this paper is to present the criteria adopted while developing the method, in addition to the minimum requirements suggested for this product.

Keywords: Plastic drinking-glass, PS, PP, one-way, minimum requirement, crush properties.

\section{Introdução}

Os copos plásticos descartáveis disponíveis no mercado brasileiro foram tendo sua massa reduzida ao longo dos anos, o que levou à necessidade de se usar dois copos para desempenhar a função de contenção de líquidos. Ou seja, uma manga (embalagem de plástico flexível utilizada para $o$ acondicionamento de copos na venda no varejo) com cem copos desempenhava a função de cinqüenta copos. Consequentemente, ocorreu o surgimento de muitas reclamações no PROCON com relação a este produto.

Assim, visando recuperar a qualidade dos copos plásticos descartáveis e evitar o desperdício de material, em 2001/2002 o Instituto Nacional do Plástico - INP solicitou um estudo de avaliação do desempenho físico-mecânico de copos plásticos descartáveis de diversas capacidades volumétricas, fabricados com massas diversas, com a finalidade de gerar subsídios aos trabalhos de padronização do "Grupo de Estudo para Normalização de Copos Descartáveis" coordenado pelo INP. Este Grupo tinha por objetivo estabelecer uma massa mínima para cada capacidade volumétrica de copo plástico descartável, além de gerar parâmetros para especificação técnica deste produto, que serviram de base para a elaboração da norma ABNT NBR 14865 Copos plásticos descartáveis ${ }^{[1]}$.
Uma vez que não foi identificada nenhuma norma técnica semelhante em outros países, foi desenvolvida uma metodologia para correlacionar o desempenho físico-mecânico dos copos plásticos descartáveis com seu desempenho durante o uso.

Este artigo tem por objetivo apresentar os critérios adotados durante o desenvolvimento desta metodologia, bem como os requisitos mínimos sugeridos para este produto.

\section{Experimental}

\section{Materiais}

Este trabalho incluiu a avaliação do desempenho físicomecânico de 96 amostras distintas de copos plásticos descartáveis, de PS e PP, através das seguintes atividades:

- Caracterização dos copos quanto a massa, a fim de trabalhar com amostras com elevado grau de homogeneidade quanto a massa;

- Análise sensorial dos copos quanto a adequação ao manuseio; e

- Determinação da resistência à compressão lateral de copos de diversas capacidades volumétricas, fabricados com massas diferentes, a saber:

- Copos de $50 \mathrm{~mL}(0,60 ; 0,65 ; 0,70 ; 0,75$; $0,80 \mathrm{~g}$ );

Autor para correspondência: Leda Coltro, CETEA - ITAL, Av. Brasil 2880, Caixa Postal 139, CEP: 13070-178, Campinas, SP, Brasil. E-mail: ledacolt@ital.sp.gov.br 
- Copos de $80 \mathrm{~mL}(1,2 ; 1,3 ; 1,4 ; 1,5$ e $1,6 \mathrm{~g})$;

- Copos de $110 \mathrm{~mL}(1,6 ; 1,7 ; 1,8 ; 1,9$ e $2,0 \mathrm{~g})$;

- Copos de $200 \mathrm{~mL}(1,80,2,00,2,20,2,40,2,60 \mathrm{e}$ 2,80 g);

- Copos de $300 \mathrm{~mL}(2,80,3,00,3,20,3,40,3,60 \mathrm{e}$ $3,80 \mathrm{~g})$

- Copos de $400 \mathrm{~mL}(4,4 ; 4,7 ; 5,0 ; 5,3$ e 5,6 g);

- Copos de $500 \mathrm{~mL}(5,5 ; 5,9 ; 6,3 ; 6,7$ e $7,1 \mathrm{~g})$; e

- Copos de $770 \mathrm{~mL}(12 ; 13 ; 14 ; 15$ e $16 \mathrm{~g})$.

A maioria dos copos de PS apresentava uma camada coextrusada de PS cristal externo como camada de brilho.

Com a finalidade de se avaliar o efeito do design do copo, foram avaliados diferentes tipos de copos: estriados, semiestriados, lisos e copos com 3,4 ou 5 anéis próximos à boca do copo. Os diferentes tipos de copo foram fornecidos por empresas distintas.

\section{Métodos}

As embalagens foram condicionadas por pelo menos 40 horas à temperatura de $23 \pm 2{ }^{\circ} \mathrm{C}$ e os ensaios conduzidos nas mesmas condições de condicionamento ${ }^{[2]}$.

\section{Caracterização dos copos quanto à massa}

Os copos foram caracterizados quanto a massa empregando-se uma balança analítica Mettler AT 400, com resolução de $0,0001 \mathrm{~g}$.

\section{Análise sensorial de adequação ao manuseio}

Os copos foram submetidos a uma análise sensorial de adequação ao manuseio através da avaliação de conjuntos de copos de diversas massas para cada capacidade volumétrica e cada design (estriado, semi-estriado, liso ou com 3 ou 4 anéis próximos à boca do copo). Cada conjunto de copos foi avaliado separadamente por um painel formado por 10 provadores. Os copos foram classificados quanto à adequação ao manuseio de acordo com a seguinte escala: Inadequado; Mínimo aceitável; Desejável; Adequado e Super dimensionado. A fim de simular a situação real de uso, os copos foram cheios com água, conforme indicado na Tabela 1 .

Tabela 1. Volume de água adicionado aos copos na avaliação sensorial de adequação ao manuseio.

\begin{tabular}{cc}
\hline Volume do copo $(\mathbf{m L})$ & Volume de água $(\mathbf{m L})$ \\
\hline 50 & $35^{*}$ \\
80 & $60^{*}$ \\
110 & $85^{*}$ \\
200 & 140 \\
300 & 230 \\
400 & 300 \\
500 & 400 \\
770 & 620 \\
\hline
\end{tabular}

*Água quente, simulando café, chá, etc.

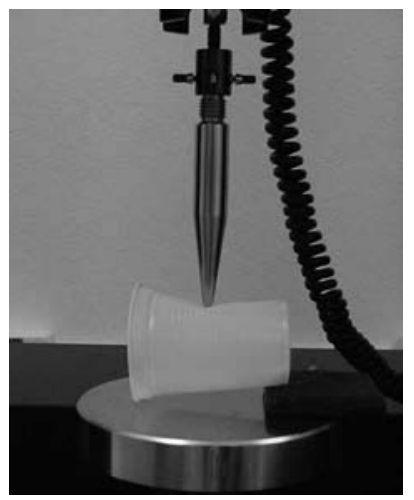

Figura 1. Resistência à compressão lateral.

\section{Resistência à compressão lateral}

Os copos foram caracterizados quanto à resistência à compressão dinâmica a partir de adaptações feitas à metodologia descrita na norma ASTM D 2659-95 (2005) - Standard test method for column crush properties of blown thermoplastic containers ${ }^{[3]}$, em um dinamógrafo de tração marca INSTRON, modelo 5500R, operando com velocidade constante de $25 \mathrm{~mm} / \mathrm{min}$ e célula de carga de $10 \mathrm{~N}$.

Para a determinação da resistência dos copos à compressão lateral, os copos foram posicionados deitados sobre a base do dinamógrafo de tração, tendo um apoio em sua base de modo que o plano da boca dos copos ficasse perpendicular à base do equipamento. A deformação foi aplicada a $15 \mathrm{~mm}$ da boca dos copos de 50, 80 e $110 \mathrm{~mL}$ e na metade da altura dos copos de demais volumes, com o auxílio de uma ponteira metálica de 6,2 mm de diâmetro, até um deslocamento limite de $10 \mathrm{~mm}$ (Figura 1). Como resultado adotou-se a resistência máxima obtida até este deslocamento.

As metodologias adotadas estão detalhadamente descritas na publicação de Alves et al. "Ensaios para avaliação de embalagens plásticas rígidas" $(1998)^{[4]}$.

\section{Resultados e Discussão}

Como a padronização exige conhecimento do desempenho dos copos, este estudo se baseou inicialmente em uma análise sensorial de adequação ao manuseio para definição de massas mínimas aceitáveis para cada capacidade volumétrica, com posterior caracterização dos copos quanto à resistência à compressão.

\section{Caracterização dos copos}

Observou-se heterogeneidade dos lotes de copos recebidos para ensaio. Por isso, foi feita uma seleção prévia dos copos de modo a gerar amostras com valor de coeficiente de variação reduzido. A Tabela 2 apresenta os resultados obtidos na caracterização destes copos previamente selecionados quanto a massa. 
Tabela 2. Caracterização dos copos de PS e PP avaliados quanto a massa (g)*.

\begin{tabular}{|c|c|c|c|}
\hline Massa (g) & $\mathbf{M}$ & IV & CV $(\%)$ \\
\hline \multicolumn{4}{|c|}{ Copos de $50 \mathrm{~mL}^{\mathrm{a}}$} \\
\hline \multicolumn{4}{|c|}{ PS - Semi-estriados } \\
\hline 0,60 & 0,606 & $0,594-0,613$ & 0,92 \\
\hline 0,65 & 0,650 & $0,640-0,657$ & 0,92 \\
\hline 0,70 & 0,702 & $0,691-0,710$ & 0,90 \\
\hline 0,75 & 0,749 & $0,742-0,759$ & 0,77 \\
\hline 0,80 & 0,802 & $0,792-0,816$ & 0,97 \\
\hline \multicolumn{4}{|c|}{ PS - Estriados } \\
\hline 0,60 & 0,602 & $0,591-0,613$ & 1,07 \\
\hline 0,65 & 0,653 & $0,642-0,665$ & 1,05 \\
\hline 0,70 & 0,703 & $0,692-0,713$ & 0,90 \\
\hline 0,75 & 0,749 & $0,740-0,759$ & 0,79 \\
\hline 0,80 & 0,805 & $0,790-0,820$ & 1,02 \\
\hline \multicolumn{4}{|c|}{ Copos de $80 \mathrm{~mL}^{\mathrm{a}}$} \\
\hline \multicolumn{4}{|c|}{ PS - Estriados } \\
\hline 1,2 & 1,183 & $1,143-1,258$ & 3,47 \\
\hline 1,3 & 1,261 & $1,267-1,347$ & 1,86 \\
\hline 1,4 & 1,409 & $1,352-1,454$ & 2,81 \\
\hline 1,5 & 1,501 & $1,455-1,544$ & 1,15 \\
\hline 1,6 & 1,610 & $1,569-1,651$ & 1,38 \\
\hline \multicolumn{4}{|c|}{ PS - 5 anéis próximos à boca do copo } \\
\hline 1,2 & 1,220 & $1,143-1,258$ & 2,54 \\
\hline 1,3 & 1,310 & $1,279-1,356$ & 1,65 \\
\hline 1,4 & 1,393 & $1,355-1,451$ & 1,54 \\
\hline 1,5 & 1,516 & $1,457-1,564$ & 1,92 \\
\hline 1,6 & 1,596 & $1,555-1,663$ & 2,28 \\
\hline
\end{tabular}

Copos de 110 mL $^{\mathrm{a}}$

PS - 3 anéis próximos à boca do copo

$\begin{array}{llll}1,6 & 1,551 & 1,540-1,571 & 0,42 \\ 1,7 & 1,725 & 1,674-1,768 & 1,78 \\ 1,8 & 1,810 & 1,792-1,829 & 0,60 \\ 1,9 & 1,903 & 1,876-1,918 & 0,61 \\ 2,0 & 1,995 & 1,982-2,018 & 0,58\end{array}$

PS - 4 anéis próximos à boca do copo

\begin{tabular}{llll}
1,6 & 1,619 & $1,590-1,648$ & 1,07 \\
1,7 & 1,703 & $1,653-1,746$ & 2,17 \\
1,8 & 1,802 & $1,760-1,831$ & 1,04 \\
1,9 & 1,908 & $1,856-1,944$ & 1,19 \\
2,0 & 2,014 & $1,990-2,048$ & 0,91 \\
\hline
\end{tabular}

Copos de 200 mL $^{\mathrm{a}}$

PS - Estriados

\begin{tabular}{llll}
2,0 & 2,058 & $1,988-2,094$ & 1,50 \\
2,2 & 2,236 & $2,199-2,279$ & 1,05 \\
2,4 & 2,468 & $2,391-2,500$ & 1,04 \\
2,6 & 2,613 & $2,517-2,682$ & 1,20 \\
\hline
\end{tabular}

\begin{tabular}{cccc}
\hline Massa $(\mathbf{g})$ & $\mathbf{M}$ & $\mathbf{I V}$ & $\mathbf{C V}(\%)$ \\
\hline \multicolumn{2}{c}{ PS - Semi-estriados } & & \\
1,8 & 1,791 & $1,683-1,905$ & 3,15 \\
2,0 & 1,955 & $1,869-2,095$ & 2,85 \\
2,2 & 2,208 & $2,110-2,297$ & 2,43 \\
2,4 & 2,422 & $2,304-2,508$ & 2,07 \\
2,6 & 2,598 & $2,503-2,700$ & 1,65 \\
PS - Lisos & & & \\
1,8 & 1,793 & $1,700-1,882$ & 3,17 \\
2,0 & 1,983 & $1,844-2,105$ & 3,32 \\
2,2 & 2,198 & $2,103-2,297$ & 2,49 \\
2,4 & 2,429 & $2,307-2,510$ & 2,57 \\
2,6 & 2,600 & $2,510-2,679$ & 1,76 \\
2,8 & 2,774 & $2,707-2,880$ & 1,60 \\
\hline
\end{tabular}

Copos de $300 \mathbf{~ m L}^{\mathrm{a}}$

PS - Estriados

$\begin{array}{llll}3,0 & 3,044 & 2,993-3,097 & 0,92 \\ 3,2 & 3,247 & 3,165-3,299 & 0,90 \\ 3,4 & 3,452 & 3,378-3,498 & 0,77 \\ 3,6 & 3,610 & 3,502-3,689 & 1,50\end{array}$

PS - Semi-estriados

$\begin{array}{llll}3,0 & 3,033 & 2,986-3,057 & 0,72 \\ 3,2 & 3,205 & 3,161-3,249 & 0,91 \\ 3,4 & 3,405 & 3,351-3,456 & 1,17 \\ 3,6 & 3,600 & 3,552-3,648 & 0,92\end{array}$

PS - Lisos

\begin{tabular}{llll}
3,0 & 3,045 & $2,951-3,192$ & 2,12 \\
3,2 & 3,169 & $3,107-3,269$ & 1,21 \\
3,4 & 3,363 & $3,229-3,492$ & 1,72 \\
3,6 & 3,584 & $3,504-3,707$ & 1,50 \\
3,8 & 3,768 & $3,703-3,858$ & 1,09 \\
\hline
\end{tabular}

Copos de $400 \mathrm{~mL}^{\mathrm{b}}$

PS - Lisos

$\begin{array}{llll}4,4 & 4,447 & 4,405-4,509 & 0,92 \\ 4,7 & 4,751 & 4,675-4,829 & 1,05 \\ 5,0 & 5,105 & 4,997-5,315 & 2,36 \\ 5,3 & 5,351 & 5,210-5,409 & 0,85 \\ 5,6 & 5,646 & 5,615-5,688 & 0,50\end{array}$

PP - Estriados

\begin{tabular}{llll}
4,4 & 4,476 & $4,440-4,518$ & 0,52 \\
4,7 & 4,748 & $4,711-4,785$ & 0,52 \\
5,0 & 5,037 & $5,003-5,073$ & 0,48 \\
5,3 & 5,266 & $5,202-5,330$ & 0,83 \\
5,6 & 5,629 & $5,601-5,668$ & 0,39 \\
\hline
\end{tabular}


Tabela 2. Continuação...

\begin{tabular}{|c|c|c|c|}
\hline Massa (g) & $\mathbf{M}$ & IV & $\mathrm{CV}(\%)$ \\
\hline \multicolumn{4}{|c|}{ Copos de $500 \mathrm{~mL}^{\mathrm{b}}$} \\
\hline \multicolumn{4}{|c|}{ PS - Lisos } \\
\hline 5,5 & 5,552 & $5,499-5,595$ & 0,58 \\
\hline 5,9 & 5,933 & $5,844-5,984$ & 0,84 \\
\hline 6,3 & 6,348 & $6,286-6,408$ & 0,62 \\
\hline 6,7 & 6,721 & $6,647-6,777$ & 0,53 \\
\hline 7,1 & 7,123 & $7,056-7,194$ & 0,69 \\
\hline \multicolumn{4}{|c|}{ PP - Estriados } \\
\hline 5,5 & 5,560 & $5,508-5,594$ & 0,49 \\
\hline 5,9 & 5,933 & $5,908-5,982$ & 0,40 \\
\hline 6,3 & 6,373 & $6,340-6,390$ & 0,24 \\
\hline 6,7 & 6,758 & $6,709-6,785$ & 0,38 \\
\hline 7,1 & 7,153 & $7,118-7,200$ & 0,43 \\
\hline \multicolumn{4}{|c|}{ Copos de 770 mL $^{b}$} \\
\hline \multicolumn{4}{|c|}{ PS - Lisos } \\
\hline 12,0 & 12,095 & $11,967-12,318$ & 1,01 \\
\hline 13,0 & 13,159 & $12,985-13,318$ & 0,85 \\
\hline 14,0 & 14,202 & $14,003-14,977$ & 1,96 \\
\hline 15,0 & 15,093 & $15,004-15,210$ & 0,48 \\
\hline 16,0 & 16,139 & $15,968-16,321$ & 0,73 \\
\hline \multicolumn{4}{|l|}{ PP - Lisos } \\
\hline 12,0 & 12,076 & $12,003-12,135$ & 0,36 \\
\hline 13,0 & 13,100 & $13,015-13,280$ & 0,63 \\
\hline 14,0 & 14,169 & $14,033-14,258$ & 0,56 \\
\hline 15,0 & 15,110 & $15,006-15,171$ & 0,33 \\
\hline 16,0 & 16,149 & $16,030-16,265$ & 0,50 \\
\hline
\end{tabular}

*Média referente a: a $30 \mathrm{e}^{\text {b }} 20$ determinações. M, IV, CV = média, intervalo de variação e coeficiente de variação, respectivamente.

\section{Análise sensorial de adequação ao manuseio}

Este ensaio teve por objetivo avaliar comparativamente o desempenho de copos de cada uma das capacidades volumétricas estudadas durante o manuseio, uma vez que o objetivo do Grupo de Estudos do Instituto Nacional do Plástico era estabelecer massas mínimas para copos plásticos descartáveis de diversas capacidades volumétricas.

Para tanto, o painel de provadores foi treinado e orientado a classificar os copos considerando o desempenho mínimo aceitável à sua função e não o melhor desempenho. Isso porquê o melhor desempenho certamente levaria à seleção do copo de maior massa e não da massa mínima do copo, como era o objetivo do estudo. Além disso, do ponto de vista ambiental, os produtos (copos, embalagens, tintas, etc.) devem desempenhar sua função com o mínimo de massa possível, conceito conhecido como redução na fonte, de modo que também é gerado menos resíduo ${ }^{[5]}$, o que se aplica perfeitamente aos copos plásticos descartáveis.

Também foi feita orientação ao painel de provadores para que o manuseio dos copos de 50, 80 e $110 \mathrm{~mL}$ fosse feito logo abaixo da boca dos mesmos, evitando-se assim que a maior espessura da borda influenciasse os resultados obtidos. No caso dos demais copos, o manuseio foi feito na metade da altura dos mesmos conforme situação normal de uso.

No caso dos copos para consumo de bebidas quentes (50, 80 e $110 \mathrm{~mL}$ ), considerou-se a tendência de atribuição de adequação ao manuseio dos copos segundo a escala utilizada e focou-se na tendência de maior atribuição percentual para as classificações "Desejável" e "Adequado" por se tratar do consumo de líquidos quentes.

Como pode ser observado na Tabela 3, o painel de provadores conseguiu perceber diferença na adequação ao manuseio dos diversos copos avaliados, sendo que houve uma tendência de maior atribuição percentual no sentido de inadequado para super dimensionado a medida que as massas dos copos aumentava, com exceção dos seguintes copos/massas:

- Copo de $50 \mathrm{~mL}$ Estriado, com 0,75 g;

- Copo de $110 \mathrm{~mL}$ com 3 anéis próximos à boca do copo, com 1,6 g; e

- Copo de $110 \mathrm{~mL}$ com 4 anéis próximos à boca do copo, com 1,9 g.

Porém, a dispersão dos resultados para os copos especificados acima não impossibilitou que se estimasse as massas mínimas. Assim, a partir da análise sensorial de adequação ao manuseio dos copos avaliados pode-se sugerir as seguintes massas mínimas para os copos:

- Copos de $50 \mathrm{~mL}: 0,7 \mathrm{~g}$;

- Copos de 80 mL: 1,4 g; e

- Copos de $110 \mathrm{~mL}: 1,8 \mathrm{~g}$.

A Tabela 4 apresenta os resultados obtidos na avaliação do manuseio dos diversos tipos de copo para bebidas frias. Para estes copos, considerou-se a tendência de atribuição de adequação ao manuseio dos copos segundo a escala utilizada e focou-se na tendência de maior atribuição percentual para as classificações "Mínimo Aceitável" e "Desejável" por se tratar de consumo de líquidos frios.

Como pode ser observado na Tabela 4, o painel de provadores conseguiu perceber diferença na adequação ao manuseio para os diversos copos avaliados, sendo que houve uma tendência de maior atribuição percentual no sentido de inadequado para super dimensionado a medida que as massas dos copos aumentava, com exceção dos seguintes copos/massas:

- Copo de $400 \mathrm{~mL}$ de PS Liso, com 5,6 g; e

- Copo de $500 \mathrm{~mL}$ de PS Liso, com 6,7 g.

No entanto, a dispersão dos resultados para os copos especificados acima não impossibilitou que se estimasse as massas mínimas para os copos avaliados. Assim, a partir da análise sensorial de adequação ao manuseio dos copos avaliados pode-se sugerir as seguintes massas mínimas para os copos plásticos:

- Copos de $200 \mathrm{~mL}: 2,2 \mathrm{~g}$;

- Copos de $300 \mathrm{~mL}: 3,2$ a 3,4 g.

- Copos de $400 \mathrm{~mL}: 5,0$ a 5,3 g;

- Copos de $500 \mathrm{~mL}: 5,9$ a 6,3 g; e

- Copos de $770 \mathrm{~mL}: 13$ a $14 \mathrm{~g}$. 
Tabela 3. Percepção sensorial de adequação ao manuseio de copos para bebidas quentes $(\%)$ *

\begin{tabular}{cccccc}
\hline Massa $(\mathrm{g})$ & \multicolumn{5}{c}{ Classificação } \\
\cline { 2 - 6 } & A & B & C & D & E \\
\hline Copos de 50 mL & & & & \\
PS - Semi-estriados & & & & \\
0,60 & $\mathbf{6 0}$ & 40 & - & - & - \\
$\mathbf{0 , 6 5}$ & 30 & $\mathbf{5 0}$ & 10 & 10 & - \\
$\mathbf{0 , 7 0}$ & - & - & $\mathbf{5 0}$ & 40 & 10 \\
$\mathbf{0 , 7 5}$ & - & - & 30 & $\mathbf{6 0}$ & 10 \\
$\mathbf{0 , 8 0}$ & - & 30 & 30 & $\mathbf{4 0}$ & - \\
PS - Estriados & & & & & \\
$\mathbf{0 , 6 0}$ & $\mathbf{4 0}$ & $\mathbf{4 0}$ & 20 & - & - \\
$\mathbf{0 , 6 5}$ & 10 & $\mathbf{4 0}$ & $\mathbf{5 0}$ & - & - \\
$\mathbf{0 , 7 0}$ & - & - & $\mathbf{7 0}$ & 30 & - \\
$\mathbf{0 , 7 5}$ & - & $\mathbf{3 0}$ & $\mathbf{3 0}$ & $\mathbf{4 0}$ & - \\
$\mathbf{0 , 8 0}$ & - & - & - & $\mathbf{8 0}$ & 20 \\
\hline
\end{tabular}

Copos de $80 \mathrm{~mL}$

PS - Estriados

$\begin{array}{cccccc}\mathbf{1 , 2} & \mathbf{3 0} & \mathbf{4 0} & 20 & 10 & - \\ \mathbf{1 , 3} & - & \mathbf{7 0} & 10 & 20 & - \\ \mathbf{1 , 4} & - & - & \mathbf{6 0} & 20 & 20 \\ \mathbf{1 , 5} & - & - & 20 & \mathbf{6 0} & 20 \\ \mathbf{1 , 6} & - & - & 10 & \mathbf{4 0} & \mathbf{5 0}\end{array}$

PS - 5 anéis próximos à boca do copo

$\begin{array}{cccccc}\mathbf{1 , 2} & \mathbf{5 0} & \mathbf{4 0} & - & 10 & - \\ \mathbf{1 , 3} & 20 & \mathbf{5 0} & 20 & - & 10 \\ \mathbf{1 , 4} & - & 10 & \mathbf{7 0} & 10 & 10 \\ \mathbf{1 , 5} & - & 10 & - & \mathbf{7 0} & 20 \\ \mathbf{1 , 6} & - & - & - & \mathbf{9 0} & 10\end{array}$

Copos de $110 \mathrm{~mL}$

PS - 3 anéis próximos à boca do copo

$\begin{array}{cccccc}\mathbf{1 , 6} & 30 & 20 & \mathbf{5 0} & - & - \\ \mathbf{1 , 7} & 20 & \mathbf{5 0} & 30 & - & - \\ \mathbf{1 , 8} & - & 10 & \mathbf{5 0} & 40 & - \\ \mathbf{1 , 9} & - & - & \mathbf{4 0} & \mathbf{4 0} & 20 \\ \mathbf{2 , 0} & - & - & 10 & \mathbf{4 0} & \mathbf{5 0}\end{array}$

PS - 4 anéis próximos à boca do copo

$\begin{array}{cccccc}\mathbf{1 , 6} & 10 & \mathbf{6 0} & 20 & 10 & - \\ \mathbf{1 , 7} & - & 30 & \mathbf{5 0} & 20 & - \\ \mathbf{1 , 8} & - & 10 & 30 & \mathbf{6 0} & - \\ \mathbf{1 , 9} & - & \mathbf{4 0} & 10 & 20 & \mathbf{3 0} \\ \mathbf{2 , 0} & - & - & 20 & \mathbf{5 0} & 30\end{array}$

*Valores referentes às atribuições de 10 provadores; Classificação: a) Inadequado; b) Mínimo aceitável; c) Desejável; d) Adequado; e e) Super dimensionado.

A fim estabelecer um procedimento analítico para expressar os resultados obtidos na análise sensorial em termos de desempenho físico-mecânico, copos das mesmas faixas de massa para cada capacidade volumétrica foram avaliados quanto à resistência à compressão lateral.
Tabela 4. Percepção sensorial de adequação ao manuseio de copos para bebidas frias $(\%)^{*}$.

\begin{tabular}{cccccc}
\hline Massa $(\mathbf{g})$ & \multicolumn{5}{c}{ Classificação } \\
\cline { 2 - 6 } & A & B & C & D & E \\
\hline Copos de 200 mL & & & & & \\
PS - Estriados & & & & & \\
$\mathbf{2 , 0}$ & $\mathbf{4 0}$ & $\mathbf{4 0}$ & 10 & 10 & - \\
$\mathbf{2 , 2}$ & 20 & $\mathbf{3 0}$ & $\mathbf{3 0}$ & 20 & - \\
$\mathbf{2 , 4}$ & - & 10 & $\mathbf{3 0}$ & $\mathbf{3 0}$ & $\mathbf{3 0}$ \\
$\mathbf{2 , 6}$ & - & - & 20 & $\mathbf{7 0}$ & 10 \\
PS - Semi-Estriados & & & & & \\
$\mathbf{1 , 8}$ & $\mathbf{9 0}$ & 10 & - & - & - \\
$\mathbf{2 , 0}$ & 20 & $\mathbf{7 0}$ & 10 & - & - \\
$\mathbf{2 , 2}$ & 10 & $\mathbf{3 0}$ & $\mathbf{5 0}$ & 10 & - \\
$\mathbf{2 , 4}$ & - & - & 30 & $\mathbf{7 0}$ & - \\
$\mathbf{2 , 6}$ & - & - & 10 & 30 & $\mathbf{6 0}$
\end{tabular}

PS - Lisos

$\begin{array}{cccccc}\mathbf{1 , 8} & \mathbf{8 0} & 20 & - & - & - \\ \mathbf{2 , 0} & \mathbf{3 0} & \mathbf{4 0} & 20 & 10 & - \\ \mathbf{2 , 2} & 20 & \mathbf{4 0} & 20 & 20 & - \\ \mathbf{2 , 4} & 10 & 10 & \mathbf{4 0} & 20 & 20 \\ \mathbf{2 , 6} & 10 & - & 20 & \mathbf{5 0} & 20 \\ \mathbf{2 , 8} & - & - & - & 20 & \mathbf{8 0}\end{array}$

Copos de $300 \mathrm{~mL}$

PS - Estriados

$\begin{array}{cccccc}\mathbf{3 , 0} & \mathbf{4 0} & \mathbf{3 0} & 10 & 20 & - \\ \mathbf{3 , 2} & - & \mathbf{6 0} & 20 & 20 & - \\ \mathbf{3 , 4} & - & 20 & \mathbf{6 0} & 10 & 10 \\ \mathbf{3 , 6} & - & - & 10 & \mathbf{4 0} & \mathbf{5 0}\end{array}$

PS - Semi-Estriados

$\begin{array}{cccccc}\mathbf{3 , 0} & 30 & \mathbf{5 0} & 10 & - & 10 \\ \mathbf{3 , 2} & 10 & \mathbf{3 0} & \mathbf{3 0} & \mathbf{3 0} & - \\ \mathbf{3 , 4} & \mathbf{3 0} & \mathbf{4 0} & \mathbf{3 0} & - & - \\ \mathbf{3 , 6} & 30 & \mathbf{4 0} & 20 & \mathbf{1 0} & -\end{array}$

PS - Lisos

$\begin{array}{cccccc}\mathbf{3 , 0} & \mathbf{6 0} & 20 & 20 & - & - \\ \mathbf{3 , 2} & \mathbf{6 0} & 20 & 20 & - & - \\ \mathbf{3 , 4} & \mathbf{3 0} & 20 & 20 & \mathbf{3 0} & - \\ \mathbf{3 , 6} & - & 20 & 10 & \mathbf{6 0} & 10 \\ \mathbf{3 , 8} & 10 & - & 20 & \mathbf{4 0} & \mathbf{3 0}\end{array}$

Copos de $400 \mathrm{~mL}$

PS - Lisos

$\begin{array}{cccccc}\mathbf{4 , 4} & 40 & \mathbf{6 0} & - & - & - \\ \mathbf{4 , 7} & 30 & \mathbf{6 0} & - & 10 & - \\ \mathbf{5 , 0} & 10 & \mathbf{5 0} & 30 & 10 & - \\ \mathbf{5 , 3} & - & - & \mathbf{7 0} & 20 & 10 \\ \mathbf{5 , 6} & 10 & - & 20 & \mathbf{6 0} & 10\end{array}$

PP - Estriados

\begin{tabular}{cccccc}
$\mathbf{4 , 4}$ & 20 & $\mathbf{6 0}$ & 10 & 10 & - \\
$\mathbf{4 , 7}$ & 10 & $\mathbf{6 0}$ & 20 & 10 & - \\
$\mathbf{5 , 0}$ & - & 20 & $\mathbf{5 0}$ & 30 & - \\
$\mathbf{5 , 3}$ & - & 10 & $\mathbf{3 0}$ & $\mathbf{4 0}$ & 20 \\
$\mathbf{5 , 6}$ & - & - & 20 & $\mathbf{5 0}$ & $\mathbf{3 0}$ \\
\hline
\end{tabular}


Tabela 4. Continuação...

\begin{tabular}{cccccc}
\hline Massa $(\mathrm{g})$ & \multicolumn{6}{c}{ Classificação } \\
\cline { 2 - 6 } & A & B & C & D & E \\
\hline Copos de 500 mL & & & & & \\
PS - Lisos & & & & & \\
$\mathbf{5 , 5}$ & $\mathbf{6 0}$ & 30 & - & 10 & - \\
$\mathbf{5 , 9}$ & 30 & $\mathbf{5 0}$ & 10 & 10 & - \\
$\mathbf{6 , 3}$ & - & 30 & $\mathbf{6 0}$ & 10 & - \\
$\mathbf{6 , 7}$ & 10 & 20 & $\mathbf{4 0}$ & 20 & 10 \\
$\mathbf{7 , 1}$ & - & 10 & $\mathbf{4 0}$ & $\mathbf{4 0}$ & 10 \\
PP - Estriados & & & & & \\
$\mathbf{5 , 5}$ & $\mathbf{6 0}$ & 30 & 10 & - & - \\
$\mathbf{5 , 9}$ & 10 & $\mathbf{6 0}$ & 30 & - & - \\
$\mathbf{6 , 3}$ & - & 20 & $\mathbf{7 0}$ & 10 & - \\
$\mathbf{6 , 7}$ & - & 10 & 30 & $\mathbf{6 0}$ & - \\
$\mathbf{7 , 1}$ & - & - & 30 & $\mathbf{7 0}$ & - \\
\hline
\end{tabular}

Copos de $770 \mathrm{~mL}$

PS - Lisos

$\begin{array}{llllll}\mathbf{1 2 , 0} & 10 & \mathbf{5 0} & 30 & 10 & -\end{array}$

$\begin{array}{llllll}\mathbf{1 3 , 0} & - & \mathbf{3 0} & \mathbf{3 0} & 20 & 20\end{array}$

$\begin{array}{llllll}\mathbf{1 4 , 0} & - & 10 & \mathbf{5 0} & 20 & 20\end{array}$

$\begin{array}{llllll}\mathbf{1 5 , 0} & - & 10 & 30 & \mathbf{4 0} & 20\end{array}$

$\mathbf{1 6 , 0} \quad-\quad-\quad-60040$

PP - Lisos

$\begin{array}{cccccc}\mathbf{1 2 , 0} & 10 & \mathbf{5 0} & 30 & 10 & - \\ \mathbf{1 3 , 0} & 10 & \mathbf{4 0} & 20 & \mathbf{3 0} & - \\ \mathbf{1 4 , 0} & - & 20 & \mathbf{4 0} & \mathbf{4 0} & - \\ \mathbf{1 5 , 0} & - & - & 20 & \mathbf{4 0} & \mathbf{4 0} \\ \mathbf{1 6 , 0} & - & - & 10 & \mathbf{5 0} & \mathbf{4 0}\end{array}$

*Valores referentes às atribuições de 10 provadores. Classificação: a) Inadequado; b) Mínimo aceitável; c) Desejável; d) Adequado; e e) Super dimensionado.

\section{Resistência à compressão lateral}

Como a resistência à compressão lateral é o fator mais crítico no manuseio de copos, optou-se por trabalhar com os dados de resistência à compressão lateral apesar de ter sido avaliada também a resistência à compressão dos copos no sentido topo/fundo.

A resistência à compressão do copo está relacionada com a distribuição de espessura da parede e o design do copo. Os designs estriado e semi-estriado aumentam a resistência à compressão, porém não permitem a determinação da distribuição de espessura. Por este motivo, a espessura foi avaliada indiretamente por meio da variação da massa dos copos. Como o design e a massa foram considerados como variáveis do estudo, a combinação dos dois fatores foi avaliada no ensaio de resistência à compressão lateral.

Os resultados de resistência à compressão dinâmica das amostras são apresentados nas Tabelas 5 e 6 . Com o objetivo de simular uma condição real de uso, estabeleceu-se $10 \mathrm{~mm}$
Tabela 5. Resistência à compressão lateral de copos plásticos para bebidas quentes.*

\begin{tabular}{|c|c|c|c|c|}
\hline \multirow{2}{*}{$\begin{array}{c}\text { Massa } \\
(\mathrm{g})\end{array}$} & \multicolumn{3}{|c|}{ Força máxima $(\mathbf{N})$} & \multirow{2}{*}{$\begin{array}{c}\text { Força/massa } \\
(\mathrm{N} / \mathrm{g})\end{array}$} \\
\hline & $\mathbf{M}$ & IV & CV (\%) & \\
\hline \multicolumn{5}{|c|}{ Copos de $50 \mathrm{~mL}$} \\
\hline \multicolumn{5}{|c|}{ PS - Semi-Estriados } \\
\hline 0,60 & $1,12^{\mathrm{a}}$ & $0,97-1,44$ & 12,6 & 1,87 \\
\hline 0,65 & $1,29^{\mathrm{b}}$ & $1,16-1,42$ & 6,8 & 1,99 \\
\hline 0,70 & $1,72^{\mathrm{c}}$ & $1,48-2,10$ & 11,2 & 2,45 \\
\hline 0,75 & $1,92^{\mathrm{d}}$ & $1,62-2,27$ & 9,6 & 2,56 \\
\hline 0,80 & $1,84^{\mathrm{c}, \mathrm{d}}$ & $1,55-2,09$ & 9,3 & 2,31 \\
\hline \multicolumn{5}{|c|}{ PS - Estriados } \\
\hline 0,60 & $1,17^{\mathrm{a}}$ & $0,84-1,37$ & 13,4 & 1,95 \\
\hline 0,65 & $1,38^{\mathrm{b}}$ & $1,11-1,65$ & 19,6 & 2,13 \\
\hline 0,70 & $1,47^{\mathrm{b}}$ & $1,29-1,67$ & 9,1 & 2,10 \\
\hline 0,75 & $1,77^{\mathrm{c}}$ & $1,52-2,17$ & 10,1 & 2,36 \\
\hline 0,80 & $1,69^{\mathrm{c}}$ & $1,50-1,91$ & 9,3 & 2,12 \\
\hline
\end{tabular}

Copos de $80 \mathrm{~mL}$

PS - Estriados

$1,2 \quad 1,42^{\mathrm{a}} \quad 1,23-1,67 \quad 11,4 \quad 1,18$

$1,3 \quad 1,90^{\mathrm{b}} \quad 1,68-2,36 \quad 10,3 \quad 1,46$

$\begin{array}{lllll}1,4 & 2,20^{\mathrm{c}} & 1,82-2,64 & 15,3 & 1,57\end{array}$

$1,5 \quad 2,41^{\mathrm{c}} \quad 1,95-2,78 \quad 10,4 \quad 1,60$

$1,6 \quad 2,68^{\mathrm{d}} \quad 2,23-2,92 \quad 9,8 \quad 1,68$

PS - 5 anéis próximos à boca do copo

$1,2 \quad 1,17^{\text {a }} \quad 0,95-1,49 \quad 15,6 \quad 0,97$

$1,3 \quad 1,57^{\mathrm{b}} \quad 1,47-1,70 \quad 5,7 \quad 1,21$

$\begin{array}{lllll}1,4 & 2,04^{\mathrm{c}} & 1,71-2,35 & 8,8 & 1,46\end{array}$

$1,5 \quad 1,73^{\text {b }} \quad 1,60-2,23 \quad 12,1 \quad 1,15$

$\begin{array}{lllll}1,6 & 2,02^{\mathrm{c}} & 1,68-2,31 & 12,0 & 1,26\end{array}$

Copos de $110 \mathrm{~mL}$

PS $\mathbf{- 3}$ anéis próximos à boca do copo

$\begin{array}{lllll}1,6 & 1,38^{\text {a }} & 1,29-1,45 & 4,4 & 0,86 \\ 1,7 & 1,34^{\text {a }} & 1,24-1,49 & 6,7 & 0,79 \\ 1,8 & 1,55^{\text {b }} & 1,36-1,72 & 6,7 & 0,86 \\ 1,9 & 1,63^{\text {b,c }} & 1,48-1,79 & 6,4 & 0,86 \\ 2,0 & 1,68^{\mathrm{c}} & 1,53-1,81 & 5,3 & 0,84\end{array}$

PS $\mathbf{- 4}$ anéis próximos à boca do copo

$\begin{array}{rrrrr}1,6 & 1,28^{\text {a }} & 1,05-1,59 & 14,9 & 0,80 \\ 1,7 & 1,41^{\text {a }} & 1,32-1,55 & 6,0 & 0,83 \\ 1,8 & 1,58^{\text {b }} & 1,35-1,81 & 10,6 & 0,88 \\ 1,9 & 1,63^{\text {b }} & 1,41-2,02 & 11,2 & 0,86 \\ 2,0 & 1,82^{\text {c }} & 1,53-2,03 & 10,2 & 0,91\end{array}$

*Média referente a 8 determinações; $\mathrm{M}, \mathrm{IV}, \mathrm{CV}=$ média, intervalo de variação e coeficiente de variação, respectivamente; ${ }^{\text {a,b,c,d }}=$ para copos de mesma capacidade volumétrica, médias seguidas de mesma letra indicam que os resultados não diferem estatisticamente, com um grau de confiança de $95 \%$.

como o máximo de deslocamento para a determinação da resistência à compressão lateral.

Como pode ser observado nas Tabelas 5 e 6, houve grande variação dos resultados, o que se refletiu no elevado valor do coeficiente de variação (de 1 a 20\%, dependendo da capacidade volumétrica). Em todos os casos, no entanto, a resistência dos copos cresce com o aumento de massa para a amostragem analisada. 
Tabela 6. Resistência à compressão lateral de copos plásticos para bebidas frias.*

\begin{tabular}{|c|c|c|c|c|}
\hline \multirow{2}{*}{$\begin{array}{c}\text { Massa } \\
\text { (g) }\end{array}$} & \multicolumn{3}{|c|}{ Força máxima $(\mathbf{N})$} & \multirow{2}{*}{$\begin{array}{c}\text { Força/massa } \\
(\mathrm{N} / \mathrm{g})\end{array}$} \\
\hline & $\mathbf{M}$ & IV & $\mathrm{CV}(\%)$ & \\
\hline \multicolumn{5}{|c|}{ Copos de $200 \mathrm{~mL}$} \\
\hline \multicolumn{5}{|c|}{ PS - Estriados } \\
\hline 2,0 & $1,19^{\mathrm{a}}$ & $1,03-1,37$ & 9,1 & 0,58 \\
\hline 2,2 & $1,45^{\mathrm{b}}$ & $1,31-1,66$ & 8,3 & 0,65 \\
\hline 2,4 & $1,79^{\mathrm{c}}$ & $1,60-1,91$ & 6,1 & 0,72 \\
\hline 2,6 & $1,93^{\mathrm{d}}$ & $1,80-2,03$ & 4,1 & 0,74 \\
\hline \multicolumn{5}{|c|}{ PS - Semi-estriados } \\
\hline 1,8 & $0,98^{\mathrm{a}}$ & $0,76-1,16$ & 11,4 & 0,53 \\
\hline 2,0 & $1,00^{\mathrm{a}}$ & $0,67-1,12$ & 13,7 & 0,51 \\
\hline 2,2 & $1,19^{\mathrm{b}}$ & $1,13-1,30$ & 4,9 & 0,54 \\
\hline 2,4 & $1,35^{\mathrm{c}}$ & $1,03-1,64$ & 13,7 & 0,56 \\
\hline 2,6 & $1,70^{\mathrm{d}}$ & $1,55-1,81$ & 4,8 & 0,65 \\
\hline \multicolumn{5}{|c|}{ PS - Lisos } \\
\hline 1,8 & $0,65^{\mathrm{a}}$ & $0,55-0,82$ & 11,8 & 0,37 \\
\hline 2,0 & $0,84^{\mathrm{b}}$ & $0,78-0,94$ & 6,7 & 0,43 \\
\hline 2,2 & $1,13^{\mathrm{c}}$ & $1,07-1,24$ & 4,8 & 0,51 \\
\hline 2,4 & $1,33^{\mathrm{d}}$ & $1,19-1,38$ & 4,6 & 0,54 \\
\hline 2,6 & $1,33^{\mathrm{d}}$ & $1,24-1,43$ & 4,4 & 0,51 \\
\hline
\end{tabular}

\begin{tabular}{|c|c|c|c|c|}
\hline \multicolumn{5}{|c|}{ Copos de $300 \mathrm{~mL}$} \\
\hline \multicolumn{5}{|c|}{ PS - Estriados } \\
\hline 3,0 & $1,08^{\mathrm{a}}$ & $0,94-1,21$ & 8,4 & 0,35 \\
\hline 3,2 & $1,28^{\mathrm{b}}$ & $1,13-1,47$ & 9,3 & 0,39 \\
\hline 3,4 & $1,60^{c}$ & $1,43-1,99$ & 11,2 & 0,46 \\
\hline 3,6 & $1,69^{\mathrm{c}}$ & $1,20-1,89$ & 12,0 & 0,47 \\
\hline \multicolumn{5}{|c|}{ PS - Semi-Estriados } \\
\hline 3,0 & $0,83^{\mathrm{b}}$ & $0,73-0,99$ & 10,6 & 0,28 \\
\hline 3,2 & $0,85^{\mathrm{b}}$ & $0,67-1,03$ & 15,2 & 0,27 \\
\hline 3,4 & $0,77^{\mathrm{a}}$ & $0,65-0,89$ & 9,9 & 0,23 \\
\hline 3,6 & $0,87^{\mathrm{b}}$ & $0,71-1,12$ & 13,1 & 0,24 \\
\hline \multicolumn{5}{|c|}{ PS - Lisos } \\
\hline 3,0 & $0,93^{\mathrm{a}}$ & $0,79-1,06$ & 10,2 & 0,31 \\
\hline 3,2 & $0,90^{\mathrm{a}}$ & $0,80-1,01$ & 8,7 & 0,28 \\
\hline 3,4 & $1,13^{\mathrm{b}}$ & $0,88-1,35$ & 13,1 & 0,33 \\
\hline 3,6 & $1,32^{\mathrm{c}}$ & $1,19-1,48$ & 7,6 & 0,37 \\
\hline 3,8 & $1,37^{\mathrm{c}}$ & $0,89-1,62$ & 18,0 & 0,36 \\
\hline
\end{tabular}

\begin{tabular}{|c|c|c|c|c|}
\hline \multicolumn{5}{|c|}{$\begin{array}{l}\text { Copos de } 400 \mathrm{~mL} \\
\text { PS - Lisos }\end{array}$} \\
\hline 4,4 & $1,07^{\mathrm{a}}$ & $0,92-1,23$ & 9,7 & 0,24 \\
\hline 4,7 & $1,08^{\mathrm{a}}$ & $0,94-1,36$ & 11,5 & 0,23 \\
\hline 5,0 & $1,19^{\mathrm{a}}$ & $1,04-1,49$ & 10,9 & 0,24 \\
\hline 5,3 & $1,73^{\mathrm{b}}$ & $1,31-2,11$ & 18,8 & 0,33 \\
\hline 5,6 & $1,76^{\mathrm{b}}$ & $1,43-2,15$ & 13,1 & 0,32 \\
\hline \multicolumn{5}{|c|}{ PP - Estriados } \\
\hline 4,4 & $1,68^{\mathrm{a}}$ & $1,58-1,78$ & 4,5 & 0,38 \\
\hline 4,7 & $1,86^{\mathrm{b}}$ & $1,81-1,96$ & 2,7 & 0,40 \\
\hline 5,0 & $2,06^{\mathrm{c}}$ & $2,01-2,10$ & 1,3 & 0,41 \\
\hline 5,3 & $2,21^{\mathrm{d}}$ & $2,08-2,34$ & 3,3 & 0,42 \\
\hline 5,6 & $2,41^{\mathrm{e}}$ & $2,34-2,49$ & 1,8 & 0,43 \\
\hline
\end{tabular}

\begin{tabular}{ccccc}
\hline Massa & \multicolumn{3}{c}{ Força máxima (N) } & Força/massa \\
\cline { 2 - 4 }$(\mathbf{g})$ & $\mathbf{M}$ & $\mathbf{I V}$ & $\mathbf{C V}(\mathbf{\%})$ & $(\mathbf{N} / \mathbf{g})$ \\
\hline \multicolumn{2}{c}{ Copos de 500 $\mathbf{~ m L}$} & & & \\
\multicolumn{2}{c}{ PS - Lisos } & & & \\
5,5 & $0,90^{\mathrm{a}}$ & $0,80-1,16$ & 11,1 & 0,16 \\
5,9 & $0,95^{\mathrm{a}}$ & $0,85-1,16$ & 11,9 & 0,16 \\
6,3 & $1,35^{\mathrm{b}}$ & $1,19-1,50$ & 9,1 & 0,21 \\
6,7 & $1,41^{\mathrm{b}}$ & $1,19-1,61$ & 10,7 & 0,21 \\
7,1 & $1,62^{\mathrm{c}}$ & $1,31-1,93$ & 12,6 & 0,23 \\
$\mathbf{P P}-\mathbf{E s t r i a d o s}$ & & & \\
5,5 & $1,36^{\mathrm{a}}$ & $1,25-1,49$ & 4,8 & 0,25 \\
5,9 & $1,47^{\mathrm{b}}$ & $1,36-1,59$ & 4,5 & 0,25 \\
6,3 & $1,75^{\mathrm{c}}$ & $1,68-1,81$ & 2,4 & 0,28 \\
6,7 & $2,00^{\mathrm{d}}$ & $1,94-2,06$ & 2,1 & 0,30 \\
7,1 & $2,19^{\mathrm{e}}$ & $2,10-2,26$ & 2,3 & 0,31 \\
\hline Copos de & $\mathbf{7 7 0} \mathbf{~ m L}$ & & & \\
PS - Lisos & & & \\
12,0 & $2,43^{\mathrm{a}}$ & $1,99-3,28$ & 19,8 & 0,20 \\
13,0 & $2,67^{\mathrm{a}, \mathrm{b}}$ & $2,27-3,69$ & 15,4 & 0,21 \\
14,0 & $3,17^{\mathrm{c}}$ & $2,53-4,08$ & 14,5 & 0,23 \\
15,0 & $3,11^{\mathrm{b}, \mathrm{c}}$ & $2,74-3,55$ & 10,4 & 0,21 \\
16,0 & $3,55^{\mathrm{c}}$ & $2,68-5,11$ & 20,1 & 0,22 \\
PP - Estriados & & & \\
12,0 & $3,18^{\mathrm{a}}$ & $3,00-3,47$ & 4,3 & 0,27 \\
13,0 & $3,69^{\mathrm{b}}$ & $3,50-3,85$ & 3,1 & 0,28 \\
14,0 & $4,30^{\mathrm{c}}$ & $3,83-4,75$ & 6,4 & 0,31 \\
15,0 & $5,28^{\mathrm{d}}$ & $4,90-5,55$ & 3,7 & 0,35 \\
16,0 & $5,90^{\mathrm{e}}$ & $5,60-6,25$ & 4,0 & 0,37 \\
\hline
\end{tabular}

*Média referente a 10 determinações; $\mathrm{M}, \mathrm{IV}, \mathrm{CV}=$ média, intervalo de variação e coeficiente de variação, respectivamente; a,b,c,d,e $=$ para copos de mesma capacidade volumétrica, médias seguidas de mesma letra indicam que os resultados não diferem estatisticamente, com um grau de confiança de $95 \%$.

Com a finalidade de facilitar a compreensão da correlação Força máxima $v s$. Massa foi calculada a relação Força máxima/Massa vs. Massa para os diversos tipos de copos (Tabelas 5 e 6). Esta relação permite que se avalie quanto um aumento de massa se reflete na resistência à compressão.

A fim de se estabelecer um procedimento analítico para especificação dos copos, foi feita uma comparação das massas mínimas dos copos estabelecidas pela análise sensorial de adequação ao manuseio com os resultados obtidos no ensaio de resistência à compressão lateral. Esta comparação é apresentada na Tabela 7.

Com base nos resultados apresentados na Tabela 7, a especificação de copos plásticos descartáveis pode ser expressa da seguinte forma:

- Copos de $50 \mathrm{~mL}$ : massa $\geq 0,7 \mathrm{~g}$ e resistência à compressão lateral > 1,5 N;

- Copos de $80 \mathrm{~mL}$ : massa $\geq 1,4 \mathrm{~g}$ e resistência à compressão lateral > 2,0 N;

- Copos de 110 mL: massa $\geq 1,8$ g e resistência à compressão lateral $>1,6 \mathrm{~N}$.

- Copos de $200 \mathrm{~mL}$ : massa $\geq 2,2 \mathrm{~g}$ e resistência à compressão lateral $\geq 1,1 \mathrm{~N}$; 
Tabela 7. Massas mínimas identificadas na análise sensorial $v s$. resistência à compressão lateral.

\begin{tabular}{|c|c|c|c|}
\hline \multirow[t]{2}{*}{ Amostra } & \multirow{2}{*}{$\begin{array}{c}\text { Análise } \\
\text { sensorial } \\
\text { Massa } \\
\text { mínima } \\
\text { (g) }\end{array}$} & \multicolumn{2}{|c|}{$\begin{array}{c}\text { Compressão } \\
\text { lateral }\end{array}$} \\
\hline & & $\begin{array}{c}\text { Força } \\
\text { máxima } \\
(\mathrm{N}) \\
\end{array}$ & $\begin{array}{c}\text { Força/ } \\
\text { massa } \\
(\mathbf{N} / g) \\
\end{array}$ \\
\hline \multicolumn{4}{|l|}{ Copo de $50 \mathrm{~mL}$} \\
\hline PS - Semi-estriado & 0,7 & 1,72 & 2,46 \\
\hline PS - Estriado & 0,7 & 1,47 & 2,10 \\
\hline \multicolumn{4}{|l|}{ Copo de $80 \mathrm{~mL}$} \\
\hline PS - Estriado & 1,4 & 2,20 & 1,57 \\
\hline $\begin{array}{l}\text { PS - } 5 \text { anéis próximos } \\
\text { à boca do copo }\end{array}$ & 1,4 & 2,04 & 1,46 \\
\hline \multicolumn{4}{|l|}{ Copo de $110 \mathrm{~mL}$} \\
\hline $\begin{array}{l}\text { PS - } 3 \text { anéis próximos } \\
\text { à boca do copo }\end{array}$ & 1,8 & 1,55 & 0,86 \\
\hline $\begin{array}{l}\text { PS - } 4 \text { anéis próximos } \\
\text { à boca do copo }\end{array}$ & 1,8 & 1,58 & 0,88 \\
\hline \multicolumn{4}{|l|}{ Copo de $200 \mathrm{~mL}$} \\
\hline PS - Estriado & 2,2 & 1,45 & 0,65 \\
\hline PS - Semi-estriado & 2,2 & 1,19 & 0,54 \\
\hline PS - Liso & 2,2 & 1,13 & 0,51 \\
\hline \multicolumn{4}{|l|}{ Copo de $300 \mathrm{~mL}$} \\
\hline PS - Estriado & 3,2 & 1,28 & 0,39 \\
\hline PS - Semi-estriado & 3,2 & 0,85 & 0,27 \\
\hline PS - Liso & 3,4 & 1,13 & 0,33 \\
\hline \multicolumn{4}{|l|}{ Copo de $400 \mathrm{~mL}$} \\
\hline PS - Liso & $5,0-5,3$ & $1,19-1,73$ & $0,24-0,33$ \\
\hline PP - Estriado & $4,7-5,0$ & $1,86-2,06$ & $0,40-0,41$ \\
\hline \multicolumn{4}{|l|}{ Copo de $500 \mathrm{~mL}$} \\
\hline PS - Liso & $5,9-6,3$ & $0,95-1,35$ & $0,16-0,21$ \\
\hline PP - Estriado & $5,9-6,3$ & $1,47-1,75$ & $0,25-0,28$ \\
\hline \multicolumn{4}{|l|}{ Copo de $770 \mathrm{~mL}$} \\
\hline PS - Liso & $13-14$ & $2,67-3,17$ & $0,21-0,23$ \\
\hline PP - Liso & 13 & 3,69 & 0,28 \\
\hline
\end{tabular}

- Copos de $300 \mathrm{~mL}$ : massa $\geq 3,2 \mathrm{~g}$ e resistência à compressão lateral $\geq 1,1 \mathrm{~N}$.

- Copos de $400 \mathrm{~mL}$ : massa $\geq 5,0 \mathrm{~g}$ e resistência à compressão lateral > 1,2 N;

- Copos de $500 \mathrm{~mL}$ : massa $\geq 5,9 \mathrm{~g}$ e resistência à compressão lateral $>1,0 \mathrm{~N}$;

- Copos de $770 \mathrm{~mL}$ : massa $\geq 13 \mathrm{~g}$ e resistência à compressão lateral $>2,7 \mathrm{~N}$.

Os valores especificados referem-se a valores médios obtidos a partir de 10 determinações.

Os valores de resistência à compressão lateral dos copos de $300 \mathrm{~mL}$ de PS semi-estriados foram inferiores àqueles apresentados pelos copos estriados e lisos de mesma capacidade volumétrica, mostrando a influência do design no desempenho dos copos. Vale ressaltar também que detalhes de design dos copos se revelaram determinantes para a aceitação da amostra pelo provador na avaliação de adequação ao manuseio.

As Figuras 2 e 3 mostram que as massas mínimas propostas situam-se na região de mudança de inflexão das curvas,

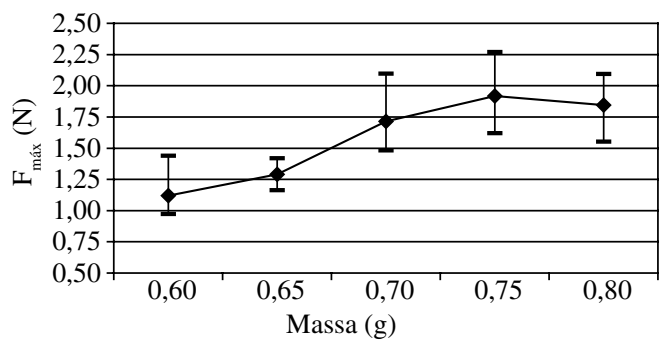

(a)

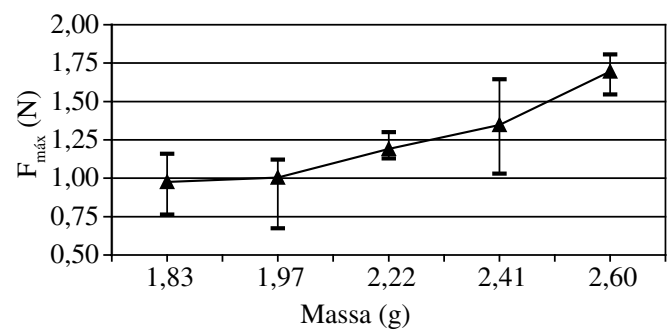

(b)

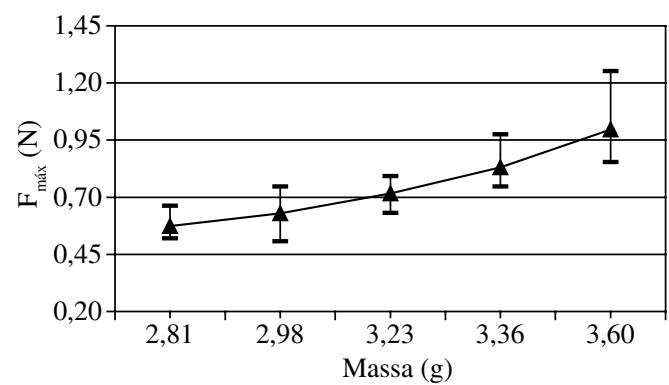

(c)

Figura 2. Resistência à compressão lateral de copos Semi-Estriados de: a) $50 \mathrm{~mL}$; b) $200 \mathrm{~mL}$; e c) $300 \mathrm{~mL}$

indicando uma alteração no desempenho das amostras. O mesmo perfil foi apresentado pelos demais copos avaliados.

\section{Conclusões}

De acordo com os resultados obtidos, pode-se concluir que:

- A análise sensorial permitiu definir as massas mínimas para que os copos plásticos descartáveis apresentem um desempenho mínimo aceitável durante o uso.

- A determinação da resistência à compressão lateral de copos plásticos descartáveis mostrou-se uma boa metodologia para a correlação da massa dos copos com seu desempenho durante o uso.

- O ensaio de resistência à compressão lateral (a $15 \mathrm{~mm}$ da boca do copo para bebidas quentes e a meia altura do copo para bebidas frias) mostrou-se mais interessante para a avaliação do desempenho fisico-mecânico dos copos do que o ensaio de resistência à compressão de topo, uma vez que simula o manuseio do copo pelo usuário final.

- A resistência à compressão lateral de copos possibilita que se diferencie copos de material e design diversos. 


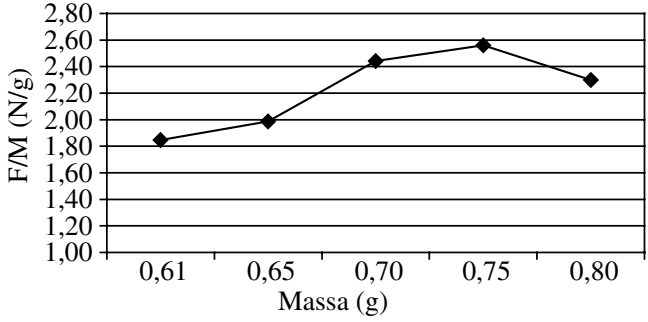

(a)

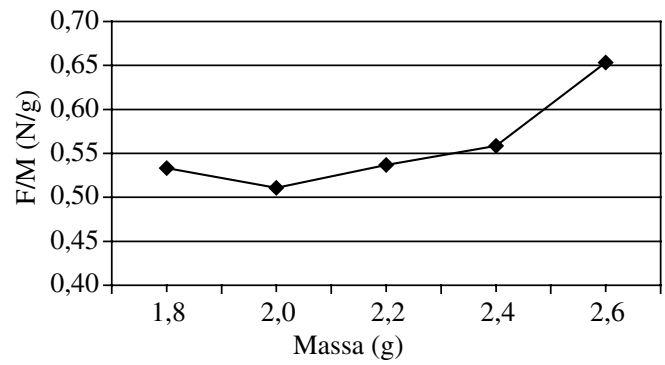

(b)

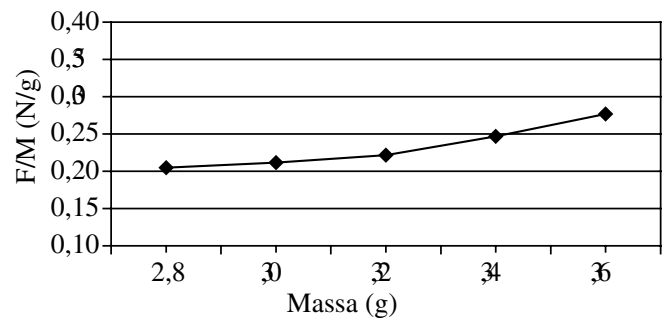

(c)

Figura 3. Relação força/massa vs. massa para a resistência à compressão lateral de copos Semi-Estriados de: a) $50 \mathrm{~mL}$; b) $200 \mathrm{~mL}$; e c) $300 \mathrm{~mL}$.

- Foi observada variabilidade das amostras de copos avaliadas neste estudo, de modo que os resultados apresentados referem-se a copos previamente selecionados para análise, de modo a se reduzir a variabilidade dos resultados.

- Mesmo com a prévia seleção das amostras, foi observada variabilidade razoável dos resultados de resistência à compressão lateral chegando-se, em alguns casos, a um coeficiente de variação da ordem de $20 \%$.

- Esta variabilidade dos resultados foi atribuída às dificuldades práticas inerentes ao processo de termoformação dos copos.
- As massas mínimas identificadas para os copos de diversas capacidades volumétricas através da análise sensorial de adequação ao manuseio segundo os critérios adotados foram correlacionados com os resultados dos ensaios de resistência à compressão lateral e permitiram o estabelecimento de requisitos mínimos para este produto em suas diversas capacidades volumétricas.

- O ensaio de resistência à compressão lateral mostrouse bastante apropriado para a avaliação do desempenho fisico-mecânico dos copos, uma vez que simula o manuseio do copo pelo usuário final.

\section{Agradecimentos}

Os autores agradecem ao Instituto Nacional do Plástico INP pelo apoio financeiro e a todos aqueles que colaboraram durante o desenvolvimento deste estudo.

\section{Referências Bibliográficas}

1. NBR 14.865 - Copos plásticos descartáveis. Rio de Janeiro, ABNT (2002).

2. ASTM. D 618-05 - "Standard practice for conditioning plastics for testing". ASTM Standard on Disc. Philadelphia, v. 08.01 (2005).

3. ASTM D 2659-95 (2005) - "Standard test method for column crush properties of blown thermoplastic containers". ASTM Standard on Disc. Philadelphia, v. 08.01 (2005).

4. Alves, R.M.V., Oliveira, L.M., Coltro, L., Garcia, E.E.C., Sarantópoulos, C.I.G.L. \& Padula, M. - "Ensaios para Avaliação de Embalagens Plásticas Rígidas”, CETEA/ ITAL, Campinas (1998).

5. Mourad, A.L., Garcia, E.E.C. \& Vilhena, A. - "Avaliação do ciclo de vida: princípios e aplicações", CETEA/ CEMPRE, Campinas (2002).

Enviado: 20/08/06

Reenviado: 06/11/06

Aprovado: 16/11/06 